\title{
Early Detection is Vital in Successful Resuscitation after Massive Mixed-air and Carbon dioxide Venous Embolism during Laparoscopic Surgery
}

\author{
Surendranath $\mathrm{V}^{1}$, Radhakrishna Patta ${ }^{2}$, Raghavendran $\mathrm{K}^{1}$
}

\section{Abstract}

Air embolism is a life threatening condition, which is commonly reported in sitting position surgery or when the venous sinuses are open. Symptoms may appear depending on volume of air entrained and rate of entrainment. Lung acts as a filter and if the area exposed is more than the critical volume, then ventilation - perfusion $(\mathrm{V} / \mathrm{Q})$ mismatch leads to hypoxia and ultimately death. In contrast, carbon dioxide in large volume, lead to right ventricular outflow tract (RVOT) obstruction and reduce cardiac output. In people with probe patent patent foramen ovale, this can reach left atrium and lead to systemic embolism. Laparoscopic surgery, in general is safe and in situations, where there is abnormal organ placement and arterio venous connection may lead to gas placement into circulation. Detection is by trans esophageal echocardiography, precordial doppler, end tidal carbon dioxide monitoring and pulse oximetry. We present one such case with altered anatomy leading to ptosis of liver and massive mixed air and carbon dioxide embolism, which was successfully re-suscitated.

Keywords : Air embolism; Carbon dioxide embolism; Laparoscopy.

Introduction

Venous air embolism (VAE) is entrainment of air into circulation due to differences in pressure gradients be-tween vascular and extravascular compartments leading to hemodynamic catastrophe with mortality ranging from $48 \%$ to $80 \%$ if undetected and untreated in elective surgeries and blunt and penetrating trauma $[1,2]$. It is very common in surgeries done on a patient in sitting position in neurosurgery, orthopedic surgery, laparoscopic surgeries, hysteroscopy and other procedures done in a position with veins exposed to air at a level higher than the heart [1].

Laparoscopic surgery is regarded to be safe as it could largely reduce mortality and morbidity. Though rare, air embolism can occur in laparoscopic surgeries due to inadvertent air placement into abdominal ves-sels, abdominal walls, or on to a solid visceral surface during laparoscopic gall bladder surgery or in patients with an abnormal anatomical vessel structure $[3,4]$. The rate and volume of air entrained will determine mortality and morbidity. Early detection is key to successful resuscitation and will improve patient survival rate. Ventilation perfusion mismatch and ultimate circulatory collapse is the mechanism of air embolism. CO2 embolus is usually due to an injection into a large artery, vein, or solid organ that is highly vascularized. A massive CO2 embolism leads to a "gas lock" at right ventricular outflow tract (RVOT) obstruction and cir-culatory collapse. This case report reiterates importance of early detection in diagnosis for a successful resuscitation even in massive air \& carbon dioxide embolism.

\section{Case report}

We present the case of a 22-year-old male (ASA II) planned for elective diagnostic laparoscopy and open congenital diaphragmatic hernia repair on the right side. He presented with symptoms of breathlessness, which was gradual in onset and progressive in nature initially with moderate exertion later to mild exertion over a period of 6-8 months. He also complained of halitosis and productive cough for which he was evaluated. On evaluation, congenital diaphragmatic hernia on right side was detected.

His chest X-ray PA view revealed right hemidiaphragm displacement and right lowerzone non-homogenous opacification. HRCT chest revealed a large defect on the right diaphragm and right lower lobe bronchiectasis. Ptosis of the liver was seen along with interspersed bowel contents between the lungs and the liver. Other blood investigations and preoperative transthoracic echocardiography (TTE), as part of a workup, were within normal limits. He was planned for diagnostic laparoscopy to define the defect and plan surgical incision for an open diaphragmatic hernia mesh repair.

$\mathrm{He}$ was attached to minimum mandatory monitors, viz., ECG, NIBP, and pulse oximetry. Central venous pressure and invasive arterial monitoring were done keeping in mind the nature of the disease and the surgical procedure that may require intraoperative and postoperative monitoring. He was induced with inj. fentanyl $2 \mathrm{mcg} / \mathrm{kg}$, inj. propofol $2 \mathrm{mg} / \mathrm{kg}$, inj. rocuronium 0.6

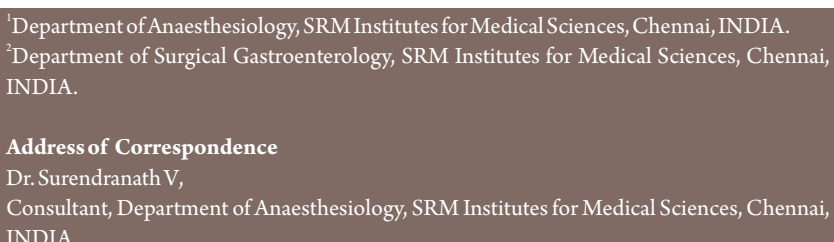

INDIA 


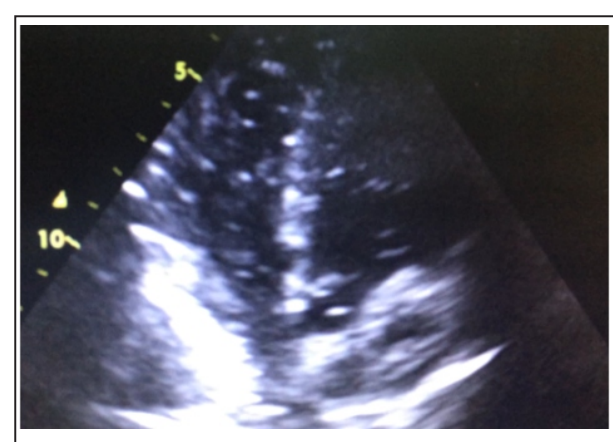

Figure 1: Transthoracic ECHO showing multiple snow flake appearance in right and left ventricle.

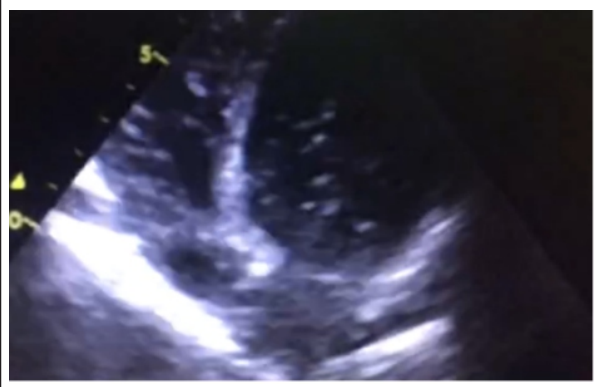

Figure 2: Transthoracic ECHO showing enlarged right ventricle suggestive of right ventricular outflow tract (RVOT) obstruction.

$\mathrm{mg} / \mathrm{kg}$, and intubated with a left-sided endobronchial double-lumen tube (32F), and position was verified using a $2.6-\mathrm{mm}$ fiber optic bronchoscope and firmly secured. Anesthesia was maintained with N2O:O2 (66\%:33\%) with end-tidal desflurane of $3 \%$ to achieve a MAC value of 0.8 .

He was placed in supine position, and Veress needle was inserted to insufflate the abdomen with $\mathrm{CO}_{2}$. The Veress needle was connected using a gas tube to a $\mathrm{CO}_{2}$ insufflator. There was a visual and audible alarm on the insufflator indicating a sudden rise in abdominal pressure; hence the gas tube was disconnected from the Veress needle and frank blood was aspirated, removing the needle immediately. This lasted for a brief period of up to 25-30 sec. At the same time, a sudden drop in end-tidal $\mathrm{CO}_{2}\left(\mathrm{Et} \mathrm{CO}_{2}\right)$ from 36 to $11 \mathrm{mmHg}$ and saturation down to $85 \%$ was noted. A provisional diagnosis of air embolism was made immediately and the patient was ventilated with $100 \%$ oxygen at a high flow $(10 \mathrm{~L} / \mathrm{min})$ in Trendelenberg position. The patient suddenly developed bradycardia and then progressed to ventricular fibrillation, for which he was resuscitated according to the ACLS protocol, and call for additional help was initiated. CPR was started and a shock of $200 \mathrm{~J}$ was delivered to treat ventricular fibrillation and continued with CPR till sinus rhythm was achieved. ROSC was restored in $<5$ minutes and was confirmed with carotid pulse and
IBP trace. Simultaneously, a 20-ml syringe was used to aspirate collected air through the central venous catheter, and approximately $60 \mathrm{ml}$ of air was aspirated. Sinus rhythm was achieved and maintained. Immediate bedside TTE was done, which showed snowflake movements representing air in all four cardiac chambers along with global hypokinesia with an EF of $30 \%$. Right atrium and right ventricle were dilated (Fig. 1, 2). In spite of a normal preoperative echo without any evidence of patent foramen ovale (PFO), air was still evident in the left side of the heart. It was decided to open the abdomen and look for any solid organ or vascular puncture. Flash laparotomy was done and a small puncture on the liver surface was noticed. Other vascular structures, like the inferior vena cava and hepatic veins, were normal. The abdomen was closed in layers. Once his vitals were stabilized, the patient was shifted to the ICU. Ventilation was continued with $80 \%$ oxygen, and he was sedated using inj. fentanyl 50 $\mathrm{mcg} / \mathrm{h}$ and inj. midazolam $2 \mathrm{mg} / \mathrm{h}$ for a further management. He was kept on inj. adrenaline $0.01 \mathrm{mcg} / \mathrm{kg}$ per minute to maintain hemodynamics, which was stopped within 1 hr. of ROSC.

In the ICU, sedation and a passive hypothermic protocol was adopted to drop temperature to $34^{\circ} \mathrm{C}$ for neuroprotection by not actively warming the patient and muscle paralysis using inj. vecuronium $6 \mathrm{mg} / \mathrm{h}$ i.v. infusion, with the patient maintained at $20^{\circ}$ Trendelenberg position. He was ventilated for $48 \mathrm{hr}$. His GCS was assessed after cessation of sedation for $>4 \mathrm{hr}$. A complete neurological examination was done, which showed a very successful resuscitation with multidisciplinary team effort with no neurological deficit. Cardiology consultation was sought in the ICU. He was started on telmisartan $20 \mathrm{mg}$ BID and metoprolol $25 \mathrm{mg}$ $\mathrm{OD}$ so as to prevent $\mathrm{LV}$ remodeling. He was weaned and extubated on the third day. $\mathrm{He}$ was observed in the ward for 4 days. His ECHO at discharge showed an improved EF of $45 \%$ from $30 \%$. He was advised to proceed to surgery after 6 months in view of a potential recurrence of the above events.

\section{Discussion}

Air embolism is a type of gas embolism, which requires a direct communication of air into the vasculature and a pressure gradient to allow air into the system. Historically, it has been reported commonly in posterior fossa surgery in Fowler's position. It has also been frequently reported during invasive vascular procedures, blunt chest injury, hysteroscopy, radical urinary bladder cystectomy, and laparoscopic procedures. Veress needle insertion into the venous system or into visceral parenchyma is a common reason during laparoscopic procedures. In severe cases, it could lead to right ventricular outflow obstruction. In adults, up to $30 \%$ have probe patent foramen Ovale (PFO) that opens when the right heart pressure increases tremendously, leading to right to left shunt, and the entrapped air passes to systemic circulation, embolizing any possible vital organ. This can manifest as stroke, limb ischemia, splenic infarction, etc. In this case, snowflake movements in all four chambers of heart suggested presence or abnormal opening of PFO, though clinically patient did not show any evidence of systemic embolism. A majority of VAEs are subclinical and hence undetected. Two factors determine severity: volume of air entrained and rate of entrainment. The volume of air that could potentially damage an organ causing mortality has been a subject of scientific scrutiny. Experiments conducted by Dion et al. on animals concluded that a volume of $<200 \mathrm{ml}$ is enough to cause cardiovascular events and $>300 \mathrm{ml}$ could cause mortality [9]. Meyer et al. predicted $60 \%$ mortality with 72 $\mathrm{ml} / \mathrm{min}$ equivalent volume of air in animals compared to humans. It was estimated that a volume of 200-300 $\mathrm{ml}$ of air can be significant in humans [5]. At a high volume, even a blood soluble gas as $\mathrm{CO} 2$ can cause a "gas lock" effect at the right ventricular outlet and increase RV afterload, pulmonary hypertension, and systemic hypotension, ultimately leading to a circulatory collapse [6]. $\mathrm{CO} 2$ infused at a rate of $1.2 \mathrm{ml} / \mathrm{kg}$ per minute can lead to catastrophe [10]. A large volume of air and sudden entrainment will obstruct pulmonary flow by increasing pulmonary vascular resistance, leading to an RVOT obstruction. This in turn could reduce pulmonary flow, LV preload, and cardiac output, leading to a circulatory collapse. An increased resistance to RV outflow will direct air embolus via right-to-left shunts to the left atrium leading to circulatory and neurological consequences [7]. Though in our patient there was no evidence of PFO by 2D TTE, trans-esophageal echocardiography (TEE) could have been more sensitive and specific in detecting PFO [8].

The rate of air entrainment is another factor that determines mortality. The alveolar 
interface provides a large reservoir to filter air entering into systemic circulation. A rapid ingress of gas $(>0.3 \mathrm{ml} / \mathrm{kg})$ could lead to a circulatory collapse. A slow accumulation of air over a long period might be less dangerous than a high volume of air entering in few seconds.

In our case, it was a mixed-air and $\mathrm{CO}_{2}$ embolism. The gas tube used to insufflate the abdomen could hold $175 \mathrm{ml}$ of water. Hence, after the Veress needle was introduced into the abdomen, approximately $175 \mathrm{ml}$ (3.5 $\mathrm{ml} / \mathrm{kg}$ in this case) of air and approximately

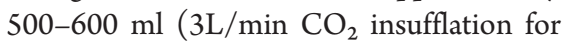
10-20 sec.) $\mathrm{CO}_{2}$ entered the circulation, leading to a circulatory catastrophe, which was detected promptly by falling $\mathrm{EtCO}_{2}$ aiding successfully resuscitation. There was high possibility that, air that preceded $\mathrm{CO}_{2}$ was inadvertently injected into circulation either through liver, caval or hepatic vein puncture, as the liver was not in anatomical position. $\mathrm{CO}_{2}$ embolism might not cause any significant hemodynamic collapse, as seen with VAE, because it is highly dissolvable and can get exhaled through the lungs. But a large volume of $\mathrm{CO}_{2}$ within a short duration may cause significant cardiopulmonary and acidbase changes, leading to significant hemodynamic changes causing outlet obstruction $[9,10]$.

$\mathrm{EtCO}_{2}$ falls dramatically in air embolism due to reduced cardiac output. Clinically, air embolism pre-sents as systemic hypotension, cyanosis, hypoxia, tachy-or bradyarrhythmia, and asystole. The most sensitive method to detect air embolism is TEE, which could detect as low as $0.1 \mathrm{ml} / \mathrm{kg}$ air. Other methods, in decreasing order of sensitivity, are transesophageal Doppler, precordial Doppler, $\mathrm{EtCO}_{2}$, pulse oximetry, and pulmonary arterial pressure (PAP). $\mathrm{EtCO}_{2}$ could detect $0.5 \mathrm{ml} / \mathrm{kg}$ air, whereas PAP might take much longer and may be least sensitive. Trans-esophageal Doppler has almost the same sensitivity as TEE (0.1 $\mathrm{ml} / \mathrm{kg}$ ), but required less-expensive equipment. The rate and severity of fall of $\mathrm{EtCO}_{2}$ predicts greater severity of hemodynamic consequences during an acute air embolism. Moreover, a fall in $\mathrm{EtCO}_{2}$ precedes fall in blood pressure [11].

Laparoscopic surgery, in comparison to open surgery is associated with less mortality and morbidity but there is a remote chance of venous air embolism to occur. Chang Seok Kim et al. reported an increased incidence of air embolism in varying grades using TEE in total laparoscopic hysterectomy (TLH) in comparison to total abdominal hysterectomy. Higher grades of air embolism are common in TLH than an open procedure, though it might not lead to a circulatory imbalance. Hence, the rate of gas entrainment is one of the prime factors for a circulatory collapse [12]. In this case, abnormal liver anatomy due to interposed bowel contents between the lungs and liver or abnormal vasculature should be factored before placing Veress needle to avoid vascular or liver puncture.

Resuscitating the patient is a team effort with a close coordination among the anesthesiologist, surgeon, and intensivist. In this case, surgery was deferred, considering patient safety, though patient was successfully resuscitated.

In conclusion, although a rare occurrence, a combined venous air and $\mathrm{CO}_{2}$ embolism during a laparoscopic surgery could become fatal if unsuspected and left untreated. The clinician should suspect early and initiate early resuscitation for a successful clinical outcome in a potentially fatal clinical complication.

\section{References}

1. Wittenberg AG, Richard AJ, Conrad SA. Venous air embolism. 2006. Available from: http://www.emedicine.com/emerg/topic787.htm.

2. Ho AM, Ling E. Systemic air embolism after lung trauma. Anesthesiology 1999; 90:564-75.

3. de Jong KIF, de Leeuw PW. Venous carbon dioxide embolism during laparoscopic cholecystectomy a T literature review. European Journal of Internal Medicine 2019; 60:9-12

4. Matteia P, Tylerb DC. Carbon dioxide embolism during laparoscopic cholecystectomy due to a patent paraumbilical vein. Journal of Pediatric Surgery 2007; 42:570-72.

5. Toung TJK, Rossberg MI, Hutchins GM. Volume of air in a lethal venous air embolism. Anesthesiology 2001; 94:360-1

6. Mirski MA, Lele AV, Fitzsimmons L, Toung TJ. Diagnosis and treatment of vascular air embolism. Anesthesiology 2007; 106:164-77.

7. Bedell EA, Berge KH, Losasso TJ. Paradoxic air embolism during venous air embolism: transesopha-geal echocardiographic evidence of transpulmonary air passage. Anesthesiology

\section{$1994 ; 80: 947-50$.}

8. Schneider B, Zienkiewicz T, Jansen V, Hofmann T, Noltenius H, Meinertz T. Diagnosis of patent fora-men ovale by transesophageal echocardiography and correlation with autopsy findings. American Journal of Cardiology 1996; 77:1202-9.

9. Dion YM, Lévesque C, Doillon CJ. Experimental carbon dioxide pulmonary embolization after vena cava laceration under pneumoperitoneum. Surgical Endoscopy 1995; 10:1065-9.

10. Mayer KL, Ho HS, Mathiesen KA, Wolfe BM. Cardiopulmonary responses to experimental venous car-bon dioxide embolism. Surgical Endoscopy 1998; 12:1025-30.

11. Kapurch CJ, Abcejo AS. The relationship between end-expired carbon dioxide tension and severity of venous air embolism during sitting neurosurgical procedures - a contemporary analysis. Journal of Clinical Anesthesia 2018; 51:49-54

12. Kim CS, Kim JY, Kwon JY, Choi SH, Na S, An J, Kim KJ. Venous air embolism during total laparoscopic hysterectomy comparison to total abdominal hysterectomy. Anesthesiology 2009; 111:50-54.

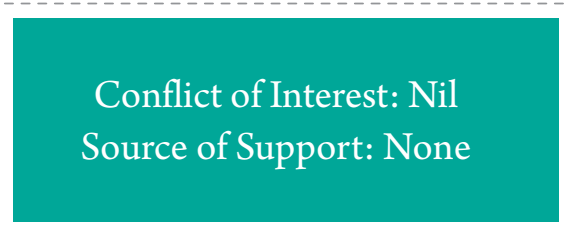

\section{How to Cite this Article}

Surendranath V, Patta R, Raghavendran K | Early Detection is Vital in Successful Resuscitation after Massive Mixed-air and Carbon dioxide Venous Embolism during Laparoscopic Surgery |Journal of Anaesthesia and Critical Care Case Reports | May-August 2020; 6(2): 14-16. 